\title{
Modelo de urbanización y desigualdad urbana. El caso de Ciudad Juárez, Chihuahua, México
}

\author{
Modelo de urbanização e desigualdade urbana. $O$ caso de \\ Ciudad Juárez, Chihuahua, México
}

\section{Urban growth model and urban inequality. The case of Ciudad Juárez, Chihuahua, Mexico}

\author{
Arehmi López García \\ https:/ / orcid.org/0000-0001-6730-2861 \\ al171807@alumnos.uacj.mx \\ Universidad Autónoma de Ciudad Juárez, UACJ, Juárez, México \\ Francisco Javier Llera \\ https:/ / orcid.org/0000-0002-0377-5835 \\ fllera@uacj.mx \\ Universidad Autónoma de Ciudad Juárez, UACJ, Juárez, México \\ Leticia Abigail Pérez Pulido \\ https:/ / orcid.org/0000-0002-2633-934X \\ al194619@alumnos.uacj.mx \\ Universidad Autónoma de Ciudad Juárez, UACJ, Juárez, México \\ Jaime Simental Orona \\ https:/ / orcid.org/0000-0002-9609-2012 \\ al194575@alumnos.uacj.mx \\ Universidad Autónoma de Ciudad Juárez, UACJ, Juárez, México
}

\begin{abstract}
Resumen: Este trabajo analiza la desigualdad urbana en la ciudad mexicana de Juárez, generada a partir de la promoción innecesaria de su expansión física por parte de los agentes públicos y privados de la localidad. La investigación enfatiza los procesos desde principios de 1990 cuando se experimentó la incorporación del sector privado en la provisión de bienes y servicios públicos. El estudio es de corte cualitativo y se apoya en el desarrollo de un estudio de caso; incluye la realización de 23 entrevistas semiestructuradas en 20 sectores habitacionales diferentes. La investigación concluye que la desigualdad urbana en Juárez tiene dos corresponsables: la política pública y la manipulación en la producción de la riqueza urbana.
\end{abstract}

Palabras clave: Mercantilización urbana, Expansión urbana, Políticas públicas.. 
Resumo: Este trabalho analisa a desigualdade urbana na cidade mexicana de Juárez, gerada a partir da promoção desnecessária de sua expansão física pelos agentes públicos e privados da localidade. A pesquisa enfatiza os processos desde o início dos anos 1990, quando se experimentou a incorporação do setor privado na provisão de bens e serviços públicos. O estudo é de natureza qualitativa e é apoiado pelo desenvolvimento de um estudo de caso, incluindo a realização de 23 entrevistas semiestruturadas em 20 setores habitacionais diferentes. A pesquisa conclui que a desigualdade urbana em Juárez possui dois fatores corresponsáveis: políticas públicas e manipulação na produção de riqueza urbana.

Palavras-chave: Mercantilização urbana, Expansão urbana, Políticas públicas.

\begin{abstract}
This paper analyzes the urban inequality in the Mexican city of Juarez, and how it is a consequence of the unnecessary urban sprawl encouraged by local public and private agents. The analysis makes emphasis on the processes experienced by the locality, since the early 1990s when the private sector became involved in the supply of public goods and services. This research work embraces a qualitative approach and develops a Case Study to provide evidence; it incorporates 23 semi-structured interviews with subjects from 20 different neighborhoods. Research results portray that the urban inequality experienced by local inhabitants in Juarez responds to two major reasons: the local public policy and the manipulation in the production of urban land.
\end{abstract}

Keywords: Urban Mercantilism, Urban Sprawl, Public policies.

\title{
INTRODUCCIÓN
}

El propósito de este trabajo es responder al cuestionamiento sobre ¿cómo el modelo de urbanización seguido en Ciudad Juárez, Chihuahua, ¿ acrecentó la desigualdad urbana entre sus habitantes? El estudio no busca discutir la desigualdad en sentido amplio sino sólo lo concerniente a la generación de la desigualdad urbana, la cual queda definida en esta investigación por los autores como el grado (nivel) de dificultad que experimenta un habitante en relación con otro(s) que coexiste(n) dentro del mismo entorno urbano para llevar a cabo la movilidad, los tiempos de traslado que experimenta, la satisfacción por el vecindario, el acceso a ambientes físicos agradables y las condiciones de paz y tranquilidad en su entorno inmediato.

En la realización de esta investigación se decidió abordar una aproximación de corte cualitativo que se apoya principalmente en la utilización de un estudio de caso sobre las fases de crecimiento urbano de Ciudad Juárez. Para enriquecer, el análisis del caso, el equipo de investigación recolectó opiniones de residentes locales para comparar su percepción sobre su vida en la ciudad y su interacción con ella. La recolección de la información se realizó a través de la aplicación de entrevistas semiestructuradas compuestas por ocho preguntas abiertas La muestra abarcó 23 juarenses que habitan en 20 sectores diferentes y que pertenecen a diferentes estratos socioeconómicos. Los datos obtenidos se incorporan al estudio mediante tablas y se discuten a través del análisis porcentual de las percepciones. Dado lo pequeño de la muestra y que el objetivo es enriquecer el planteamiento cualitativo, 
los resultados no pueden generalizarse, pero sí permitieron enriquecer la evidencia del estudio de caso.

A partir de ello, y para efectos de organización, el texto se divide en seis partes. La primera sección discute la transformación del papel del Estado y de las políticas públicas en México; la segunda aborda la mercantilización de las ciudades en el contexto mexicano; la tercera parte describe el abordaje metodológico realizado en este trabajo; la cuarta sección desarrolla el estudio de caso para describir cómo se ha dado la ocupación territorial y el grado de dificultad que experimentan algunos habitantes en su interacción diaria dentro de la ciudad; la quinta sección incluye los resultados identificados a partir de la realización de entrevistas; la sexta sección desarrolla una discusión comparando el caso de Ciudad Juárez con otras áreas urbanas del país, finalmente, la última sección se destina a conclusiones y recomendaciones.

\section{LA TRANSFORMACIÓN EN EL ACCIONAR DEL ESTADO Y LA CONFORMACIÓN DE LAS POLÍTICAS PÚBLICAS EN MÉXICO}

Uno de los grandes cambios que produjo el proceso de globalización entre los países del mundo fue la transformación en el papel del Estado. A principios de 1980, tanto en los Estados Unidos de América como en el Reino Unido, se empieza a cuestionar el papel del Estado como agente benefactor interesado en satisfacer necesidades sociales que implicaban la erogación de montos públicos considerables. Estas ideas llevaron a consolidar una nueva corriente de pensamiento interesada en limitar el tamaño y la acción del gobierno. La nueva corriente de pensamiento impulsó el desarrollo de teorías como la de la libre elección (Public Choice Theory) que establece que todo lo que el Estado no pudiera hacer y brindar en términos competitivos del mercado debería de transferirlo para su realización al sector privado (Llera y López-Nórez, 2017).

La nueva visión sobre el accionar del Estado se basaba fundamentalmente en introducir mecanismos de mercado en la prestación de servicios y otorgamiento de bienes públicos, en estimular la competencia y en impulsar la rendición de cuentas mediante el establecimiento de indicadores (Llera y López-Nórez, 2017). Esta perspectiva impulsada tanto en los Estados Unidos de América como particularmente en Inglaterra, pronto llevó a estos gobiernos a introducir programas y políticas públicas en las que los gobiernos regionales y locales deberían asumir nuevas responsabilidades ya sin apoyo del gobierno central. Este escenario también facilitó la inserción del sector privado en la prestación de diversos servicios públicos que los gobiernos locales difícilmente hubieran podido ofertar por sí mismos. Ese modelo sobre el nuevo papel del Estado fue pronto adoptado en el ámbito mundial por diferentes países en etapas distintas.

En México, y en gran parte de Latinoamérica, las nuevas reglas para el accionar del Estado empezaron a adoptarse gradualmente desde principios de 1990 bajo la concepción de reformas del Estado (Llera y López-Nórez, 2017). Progresivamente, durante ese periodo de apertura económica, el Estado mexicano empieza a apoyarse en las instituciones 
financieras y empresas privadas para atender la construcción de vivienda pública, que antaño era responsabilidad única y absoluta de instituciones de carácter gubernamental como el Instituto del Fondo Nacional de la Vivienda para los Trabajadores (INFONAVIT), el Fondo de la Vivienda del Instituto de Seguridad y Servicios Sociales de los Trabajadores del Estado (FOVISSSTE) y el Fondo Nacional de Habitaciones Populares (FONHAPO), entre otras. Un rubro complementario en que el Estado mexicano incorporó al sector privado fue en la inversión en infraestructura básica y proyectos públicos que hasta antes del año 2012 eran de responsabilidad exclusiva del sector gubernamental (Llera, 2015a).

La aprobación de la Ley de Asociaciones Público-Privadas en México, en el año 2012, vino a legitimar la plena participación de la iniciativa privada en prácticamente todo el accionar gubernamental del Estado mexicano. La ley en mención es muy importante porque literalmente adopta los principios de mercado impulsados por la teoría de la libre elección al formalizar la participación del sector privado para "prestar servicios al sector público o al usuario final - y también para que se pueda utilizar infraestructura provista total o parcialmente por el sector privado - con objetivos que aumenten el bienestar social y los niveles de inversión en el país" (Llera, 2015a, p. 102). A partir de este momento, se magnifica legítimamente la presencia del sector privado en la vida urbana de México, dado que dicho sector se avocó al desarrollo de proyectos y prestación de servicios en diversos ámbitos entre los que sobresalen la construcción y prestación de servicios en hospitales regionales, edificios gubernamentales, universidades politécnicas, puentes, obras hidráulicas y el manejo de concesiones de trenes suburbanos, autopistas y libramientos, entre otros (Llera, 2015a).

La incorporación del sector privado en el accionar del Estado no es un hecho criticable per se, sino que lo reprochable es la apariencia que ha venido adquiriendo la prestación de servicios y el acceso a bienes públicos por parte de la población mexicana en la mayoría de las ciudades. En la prestación de dichos servicios o en la oferta de bienes públicos, el objetivo central que persigue el sector privado es la obtención de ganancia o lucro, por parte de los prestadores de dichos servicios o de quienes ofrecen bienes, la forma como se han dado los procesos de privatización en el contexto mexicano y la ausencia de un Estado guardián y con contrapeso moral, a los mecanismos de mercado, han producido que un alto porcentaje de la población mexicana experimente alguna forma de desigualdad urbana. Stiglitz (2019a) lo sintetiza muy bien al establecer que la introducción de mecanismos de mercado en una sociedad no es un fin por sí mismo, sino el medio para alcanzar un fin; en este caso, debería ser lograr una sociedad más próspera.

En resumen, en México, el papel del gobierno dentro del Circuito de Bienes Raíces ha sido fundamental para generar riqueza, como argumentaban Henri Lefebvre (1972a, 1972b, 1974, 1983, 1991) y David Harvey (1990, 2009, 2012, 2014), pero también ha sido fundamental para marginar a los que menos invierten en el contexto urbano (Llera, 2015b). La política pública en los ámbitos locales mexicanos ha dado prioridad a la protección del sector inmobiliario privado y ha marginado a los sectores desarrollados en forma autogestora o a los sectores desarrollados por agentes diferentes al capital inmobiliario. A este patrón, Bullard (1993) lo ha llamado discriminación habitacional entre las colonias 
o vecindarios de una misma ciudad. La discriminación habitacional, dentro de la ciudad mexicana, es muy relevante porque empieza a convertir a la colonia o al vecindario en el que se habita, en un factor que determina la movilidad social y el éxito profesional de las personas. En otras sociedades, como la estadounidense, ya algunos autores como Stiglitz (2019b) han reconocido que crecer en el vecindario 'adecuado', está empezando a convertirse en uno de los aspectos más importantes para determinar el éxito en la vida futura de las personas. Esto, paradójicamente, es contrario a los principios de toda sociedad capitalista y democrática que se basa fundamentalmente en el principio de igualdad entre los ciudadanos (Nel-lo y Goma, 2018).

\section{LA MERCANTILIZACIÓN DE LA CIUDAD MEXICANA}

La incorporación del sector privado como acompañante en la ejecución de las políticas públicas también transformó la forma de concebir la ciudad, de realizar transacciones dentro de ella y de tomar decisiones por parte de los habitantes. La lógica de mercado empezó a reemplazar a la concepción de ciudad como un espacio de goce público e igualitario; en donde los edificios y espacios públicos constituían los principales referentes para crear identidad y crear deseabilidad de una ciudad. Por el contrario, la lógica de mercado empezó a incorporar dinámicas distintas para vivir en la ciudad una vez que aparecieron nuevos actores encargados de guiar y construir modelos de crecimiento urbano diferentes. Ese modelo no solo priorizó, dentro de la ciudad, la separación de sectores por poder de inversión; también legitimó entre la población la visión de la ciudad como mercancía. En la consecución y consolidación de esta visión fue fundamental obtener el control en el acceso al suelo urbano, controlar y dominar la construcción de vivienda de interés social y jugar con la creación de la plusvalía urbana local.

En el proceso de mercantilización de Ciudad Juárez han existido actores y dinámicas que influyen para que la ciudad incremente o reduzca su valor como un bien de cambio (Fig. 1). En primer lugar, los desarrolladores inmobiliarios son importantes para el proceso de construcción y comercialización de las propiedades dentro de las ciudades, mismos que pueden o no ser manipulados o manipular a los grupos de poder que las gobiernan. Un segundo grupo de agentes sería quienes controlan la inversión que se hace en la ciudad y el capital económico que se destina a ella. El capital económico y las inversiones pueden ser públicos o privados y su relevancia consiste en que son éstos los principales responsables del mantenimiento de las urbes y con ello de la mayor valorización o depreciación de ciertos sectores en la ciudad. La acción gubernamental a través de la zonificación y control de usos de suelo y la manipulación de los habitantes para desplazarlos hacia zonas que presentan mayores dificultades para la vida urbana en Ciudad Juárez, constituyen agentes finales que participan en el proceso de mercantilización de la ciudad y que directamente tienen incidencia en el incremento de las diferencias en el nivel de vida dentro de la ciudad, son la acción gubernamental a través de la zonificación y control de usos de suelo y la 
manipulación de los habitantes para desplazarlos hacia zonas que presentan mayores dificultades para la vida urbana en Ciudad Juárez.

Figura 1: Agentes responsables de la creación del valor en la propiedad mexicana.

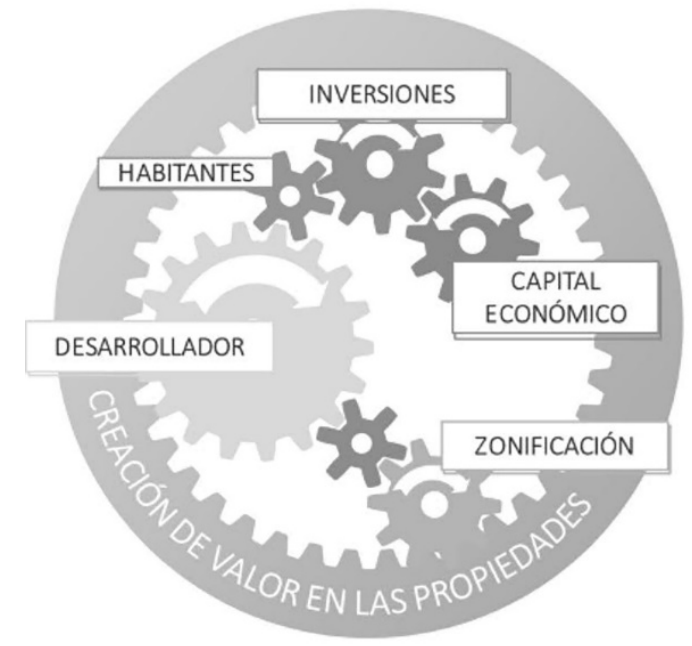

La interacción entre todos esos actores y las dinámicas que ellos impulsaron se vio reflejada en la generación de cuatro consecuencias concretas que facilitaron la mercantilización de la vida urbana en Ciudad Juárez y que se tradujeron en desigualdad urbana: 1) la especulación con el suelo urbano, 2) el aumento en la renta del suelo, 3) el cambio en las condiciones para acceder a la vivienda y 4) la discriminación habitacional por ingreso. En términos de la especulación con el suelo urbano, se puede señalar que ésta es una de las actividades que más riqueza produce dentro de las ciudades. La riqueza es generada en cada ciudad de manera diferente y en el espacio urbano esta se produce particularmente a partir de la transacción y generación de plusvalía. El suelo urbano por sí solo no tiene valor a menos que alguien ambicione invertir en él (George, 1963). En Ciudad Juárez, la especulación con el suelo urbano ha sido el factor que más ha enriquecido a un grupo reducido de acumuladores de terreno y el factor que más ha influido en la conformación de una zona urbana desarticulada entre sí y dotada de servicios públicos e infraestructura deficiente para un amplio segmento de los habitantes locales.

El aumento en la renta del suelo ha sido señalado como uno de los aspectos que mayor impacto ha tenido para generar condiciones de desigualdad urbana entre los habitantes de una localidad (Obeng-Odoom, 2015). Lo anterior debido a que durante las últimas décadas las prácticas de acumulación de riqueza en las ciudades se han centrado en buscar la obtención de ganancias a partir de la especulación con la compra y venta del suelo urbano. Como se señaló, los principales responsables de estas prácticas han sido inversionistas que han acumulado grandes extensiones de tierra, "instituciones financieras, compañías constructoras, agentes inmobiliarios y grupos políticos, entre otros" (Harvey, 2012, p. 47). La presencia de estos actores ha venido acrecentándose a medida que el suelo urbano se ha integrado gradualmente a dinámicas impulsadas por los procesos de globalización experimentados prácticamente por la mayoría de las ciudades del mundo. En Ciudad Juárez, el aumento en la renta del suelo se puede explicar a partir 
de la compra de suelo agrícola barato en la periferia, la manipulación del dinero público para la introducción de servicios y la edificación de vivienda formal para grupos de bajos ingresos con la recuperación inmediata de la inversión, a través de créditos bancarios.

El cambio en las condiciones de acceso a la vivienda ha sido otro de los elementos que en general, han contribuido al incremento de la desigualdad entre la población en las ciudades mexicanas contemporáneas y en particular en el caso de Ciudad Juárez. El hecho de que el sector gubernamental se haya separado de la construcción de vivienda pública desde la década de los noventa y que el proceso de adquisición de vivienda fuera vinculado grandemente a las instituciones financieras que otorgan créditos, ha tenido como resultado la exclusión de un alto porcentaje de la población a este bien material. A partir de que las instituciones financieras se convierten en el principal filtro para determinar quién es o no sujeto para adquirir vivienda, el proceso beneficia sólo a la población con mejor capacidad de pago, perteneciente tradicionalmente a esquemas de empleo formal. Este patrón generó dos resultados en la sociedad mexicana; por una parte, los principales beneficiarios han sido las grandes compañías constructoras que solo son intermediarios que recuperan rápidamente su inversión y que facilitan a las instituciones bancarias la reproducción de su capital a través de la ubicación de créditos bancarios mediante los compradores de vivienda y, por otra parte, las grandes perdedoras han sido las ciudades.

La última consecuencia de la mercantilización de la vida urbana de Ciudad Juárez ha sido la discriminación habitacional por ingreso. La ciudad, como una mercancía o un bien de cambio, ha desarrollado una serie de mecanismos de discriminación que están determinados por cuánto dinero puede invertir la población para habitar en ella. Este mecanismo que combina aspectos socioeconómicos y espaciales está determinado por el trabajo dentro del sector formal y por la ubicación que se tiene dentro de dicho sector. De esta manera, la diferencia de ingresos también se traduce en un mayor o menor grado de marginación dentro del espacio urbano. Por ejemplo, una persona que labora como directivo en una institución tiene más poder de inversión en la ciudad que uno que labora como oficinista. El trabajo en el sector informal, de antemano, orilla a los que laboran en él a una mayor marginación socio espacial en la ciudad. En resumen, el nivel de ingreso por familia se ha convertido en factor relevante de la ciudad mercantil para determinar qué grado de discriminación espacial experimenta un habitante dentro de ella.

Nel-lo y Goma (2018) establecen que la desigualdad es un 'fenómeno' que afecta a todos los grupos sociales y, por lo tanto, a todos los sectores residenciales dentro de cualquier ciudad. Sin embargo, es necesario señalar que aún y cuando la desigualdad afecta a todos los grupos sociales, ella se experimenta en diferentes niveles en los distintos sectores habitacionales. En Ciudad Juárez, la desigualdad urbana se ha reflejado en marginación socioespacial y esta se ha incrementado a medida que la ciudad se ha expandido físicamente. A principios de la primera década del año 2000 hubo un crecimiento exponencial no planificado al suroriente de la ciudad, que significó una expansión sin atención a los espacios urbanos para abastecer la demanda de servicios básicos necesarios para dichas comunidades (Maycotte, 2010). Esa expansión física (ver Fig. 2) que fue acompañada por la movilización encubierta de la población con más bajos ingresos, a través de la construcción 
de vivienda económica, dio pie al surgimiento de discriminación habitacional por ingreso, siendo la zona suroriente de Ciudad Juárez la más discriminada a partir de las dificultades que implica para la población de bajo ingreso vivir ahí e interactuar con el resto de la ciudad (Pérez, 2018).

Figura 2: Crecimiento urbano en Ciudad Juárez

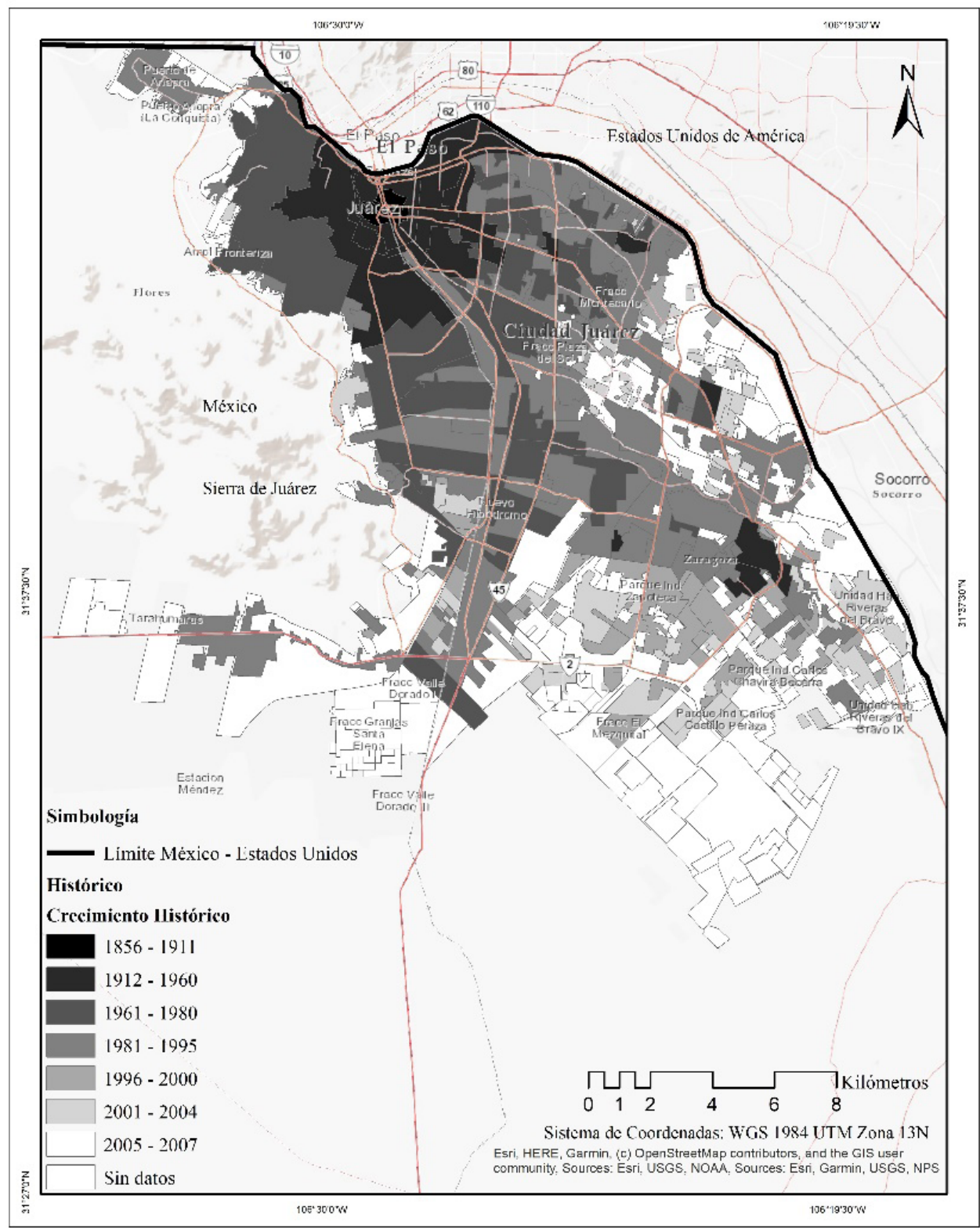

Fuente: Los autores, con datos del IMIP (2010).

La asignación de vivienda de interés social al suroriente de la ciudad, para una población de bajo poder adquisitivo en un sector alejado, modificó la dinámica urbana de la ciudad. En esta zona un porcentaje considerable de los habitantes se ubica en la llamada economía de supervivencia, con trabajos en el sector informal y cuyos ingresos son extremadamente limitados. El monto de ingreso a través del empleo formal es tan bajo que obliga a las personas a compensar sus gastos mediante prácticas informales, tales como 
la venta de automóviles usados y productos de origen estadounidense ingresados sin el pago en aduanas (fayuca), venta de productos de segunda mano o alimentos en mercados populares, comercio ambulante en espacios públicos o a través de las redes sociales, pese a este esfuerzo muchas continúan condenadas a morar en viviendas en condiciones lamentables. Aunado a la limitación de ingreso, la movilidad también se convierte en un reto enorme dado la dificultad para transportarse desde áreas alejadas a los centros de trabajo mediante el uso de transporte público, el cual además de escaso, presenta múltiples deficiencias en calidad y seguridad. Al interior de las zonas habitacionales la ausencia de áreas para el esparcimiento, la facilidad para realizar actividades criminales y el abandono del ambiente construido que las rodea, representan considerables obstáculos para el desarrollo de una vida urbana plena por parte de esa población. Estas condiciones hacen evidente la discriminación habitacional por ingreso que se experimenta en Ciudad Juárez en donde la manipulación de las políticas urbanas ha producido un lastre económico y social, y degradado los niveles de vida para los habitantes de uno de los sectores de la ciudad.

\section{CIUDAD JUÁREZ COMO CASO DE ESTUDIO}

Ciudad Juárez, Chihuahua, es una de las ciudades más antiguas en la frontera norte de México. Fundado en 1659, este asentamiento poblacional ya existía antes del establecimiento de los límites que actualmente dividen a México de los Estados Unidos. (Llera y LópezNórez, 2017). Juárez fue una localidad de poca población y de una conformación urbana pequeña por mucho tiempo, hasta que a mediados de la década de 1960 experimentó un acelerado crecimiento demográfico derivado del impulso por parte del gobierno federal al Plan de Industrialización Fronteriza (PIF). Desde entonces los volúmenes de población migrante se incrementaron con un consecuente impacto en la morfología de la ciudad (Santiago, 2013).

Una de las primeras manifestaciones de polarización o desigualdad urbana que se generaron fue el asentamiento de la población migrante en las áreas de mayor complejidad topográfica en la región y la edificación por autoconstrucción de más del cincuenta por ciento de la vivienda que existió en la ciudad entre 1960 y 1980 (López-Nórez, 2017). Ello derivado de la incapacidad del sector público mexicano de proveer de vivienda formal a un número de población que se incrementó a niveles desproporcionados durante el periodo 1960 a 1980, a la par del auge industrial en la ciudad.

Territorialmente, la población de más bajos recursos se asentó en la zona poniente y sur de la ciudad en tanto que sus fuentes de trabajo se ubicaban en el sector opuesto. Esta dicotomía dio pauta a un segundo aspecto de desigualdad urbana al forzar a casi la mitad de la población a cruzar la ciudad de lado a lado diariamente. Por otra parte, los segmentos de población de estratos socioeconómico medio y altos se concentraron en el sector norte de la ciudad, lo que les daba cercanía tanto para cruzar a los Estados Unidos de América como para acceder a las nuevas zonas comerciales, de servicios, industriales 
y de oficinas que fueron surgiendo con el paso de los años. Es decir, la movilidad de estos estratos para realizar sus actividades fue menos compleja durante ese periodo.

El crecimiento disperso de la ciudad comienza a darse a partir de la llegada de la industria maquiladora de exportación. Este tipo de industria impulsó el desarrollo de los parques industriales y con ellos contribuyó a gestar una nueva dinámica dentro del desarrollo urbano de la ciudad, validando la expansión física hacia su zona oriente. Ese proceso de expansión física que se justificaba mediante la construcción de parques industriales o plantas industriales en zonas remotas también contribuyó a desplazar - a zonas cada vez más desconectadas y sin satisfactores - a la población que migraba a la ciudad con la expectativa de obtener un empleo en la industria maquiladora (Fuentes, 2001).

A fines del año 1980 y principios del año 1990 la vida en la ciudad comienza a tomar características diferentes. El país empieza a promover la participación del sector privado en algunas de las tareas gubernamentales, particularmente, la construcción de vivienda. En el ámbito político local, esta nueva orientación del país se tradujo en erradicar el fomento clientelista de los asentamientos irregulares hacia mediados de los 90 como forma de justificar la expansión urbana, en promover el crecimiento de la ciudad hacia el sur y suroriente de la ciudad mediante compra de terrenos a los particulares, y en el surgimiento de las compañías constructoras particulares como los grandes socios de las instancias públicas para la construcción de vivienda de interés social. Esto multiplicó la aparición de fraccionamientos populares y de clase media en la zona sur de la ciudad. Dada la cercanía de estos fraccionamientos con las zonas industriales originales también se redujo, en cierto grado, el número de personas que cruzaban la ciudad de lado a lado diariamente para ir a laborar.

El periodo comprendido entre los años 2000 y 2019 se ha caracterizado por la aparición de nuevos rasgos y características de la desigualdad urbana entre la población de Ciudad Juárez. La dispersión urbana y la baja densidad poblacional (38.6 personas por hectárea, según datos de INEGI-2010) dentro del territorio urbano han sido factores importantes para crear fragilidad en la provisión de seguridad pública y facilitar la delincuencia y el vandalismo en los espacios públicos e infraestructura urbana. Es decir, a mayor distancia de las zonas consolidadas mayor deterioro del ambiente construido.

Aunado a lo anterior, se observa la apropiación de las zonas de reserva ecológica para la edificación de proyectos habitacionales y comerciales para las clases medias y altas. La construcción de vivienda en las zonas más dispersas, la manipulación de la inversión pública a través de proyectos educativos para otorgar plusvalía a terrenos privados, fueron elementos que desempeñaron un papel importante en la dispersión poblacional. Como se puede observar en la Figura 3, habitantes con ingresos mínimos, generalmente empleados en el sector industrial, se trasladaron de la zona norponiente, al suroriente de la ciudad. Este proceso generó dos nuevos tipos de desigualdad urbana. 
Figura 3. Densidad poblacional en Ciudad Juárez

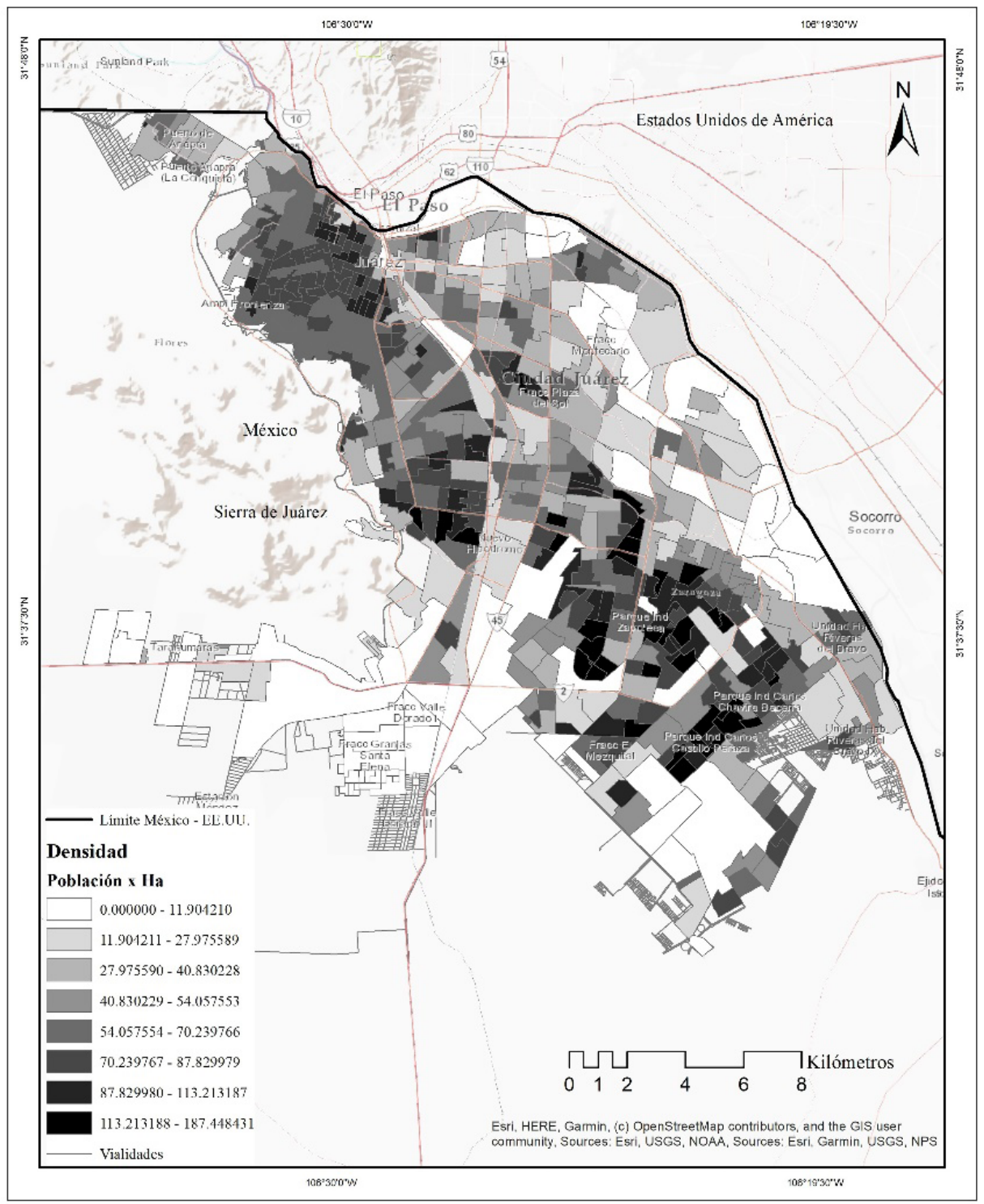

Fuente: Los autores, con datos de INEGI (2010)

Un primer tipo de desigualdad urbana o polarización entre segmentos de población es la estratificación de la movilidad. Esta situación inhibe la posibilidad de realizar actividades de recreación o entretenimiento, ya que, de manera general, se encuentran geográficamente más cercanas a los habitantes de clase media y alta. Por tanto, a mayor distancia de la zona habitacional hay menores oportunidades para la recreación. Otro tipo de desigualdad urbana es la fractura que el modelo de urbanización ha producido en el territorio. Ello ha generado la separación entre la población de adultos mayores y la población de otros grupos de edad. Esta separación entre áreas urbanas nuevas y áreas urbanas antiguas tiene implicaciones relevantes para las familias jóvenes de clase baja. Estos grupos son los que mayormente han sido relocalizados por el mercado hacia las áreas más distantes de las zonas centrales dificultando la interacción frecuente con sus padres y abuelos (LópezNórez, 2017). 
En todo este proceso de cambios por los que ha transitado la vida urbana de Ciudad Juárez, pareciera ser que la misma población local es quien menos ha concientizado la forma en que se ha estratificado o diferenciado la convivencia urbana. En la actualidad algunos habitantes locales experimentan dificultad y percepciones variadas para identificar las limitantes y diferencias en su interacción cotidiana dentro de la ciudad; particularmente en lo relacionado con la movilidad, los tiempos que utilizan para trasladarse a sus labores o actividades, la posibilidad de disfrutar la vida comunitaria en su vecindario, la posibilidad de recrearse en ambientes físicos agradables y la posibilidad de experimentar seguridad en su entorno inmediato. Todos estos elementos polarizan y marcan diferencia en la convivencia e interacción de la población juarense al interior del contexto urbano.

\section{RESULTADOS}

Algunas opiniones sobre las diferencias que se experimentan en la ciudad fueron compartidas al equipo de investigación por 23 individuos que habitan en 20 colonias o fraccionamientos diferentes y que pertenecen a diferentes estratos socioeconómicos. En esta investigación se establecieron estos estratos considerando el ingreso y profesión de la población en el contexto de las ciudades del norte de México, en particular en Ciudad Juárez donde el salario mínimo mensual al año 2020 corresponde a $\$ 5,641.024$ pesos y la fuente principal de empleo formal corresponde a la industria de la transformación con un $65.34 \%$, el comercio con un $10.69 \%$ y los servicios para las empresas, personas y el hogar con un 10.56\%, considerando que "a finales del 2018 menos del 25\% de los trabajadores formales [estaban] registrados con menos de dos salarios mínimos" (Plan Estratégico de Juárez, 2019).

Para efectos de este trabajo, se diferenciaron tres grupos según su grado de marginación ${ }^{1}$ en 1) alta marginación, correspondiente a ingresos iguales o menores a $\$ 14,999$ pesos mensuales, 2) media marginación, con ingresos en un rango de $\$ 15,000$ a $\$ 29,000$ pesos mensuales y, 3) baja marginación, con un ingreso igual o mayor a $\$ 29,999$ pesos mensuales.

Dado lo pequeño de la muestra, se entiende que los resultados no permiten generalizaciones. Sin embargo, permiten reforzar la evidencia del estudio de caso aquí analizado, que muestra cómo el proceso de urbanización ocurrido en Ciudad Juárez, acrecentó las diferencias en la interacción social-urbana local, aunado a las condiciones económicas de la población. Un primer elemento de análisis lo constituyeron las diferencias por origen de vecindario, colonia o fraccionamiento. La Figura 4 muestra el contraste entre la ubicación de los participantes en la escala de grados de marginación establecida en este

1 La marginación es definida por el Consejo Nacional de Población (CONAPO) como, un fenómeno multidimensional y estructural originado, en última instancia, por el modelo de producción económica expresado en la desigual distribución del progreso, en la estructura productiva y en la exclusión de diversos grupos sociales, tanto del proceso como de los beneficios del desarrollo (n/a, p. 11). Esta definición permite visualizar la dimensión económica preponderante en el uso del concepto de marginación, que es el enfoque utilizado en este documento. 
análisis según su ingreso y la ubicación del equipamiento, de acuerdo con las 20 zonas de origen de los participantes, la ubicación de hospitales, escuelas y parques urbanos.

Figura 4. Ubicación de participantes y equipamiento básico en Ciudad Juárez.

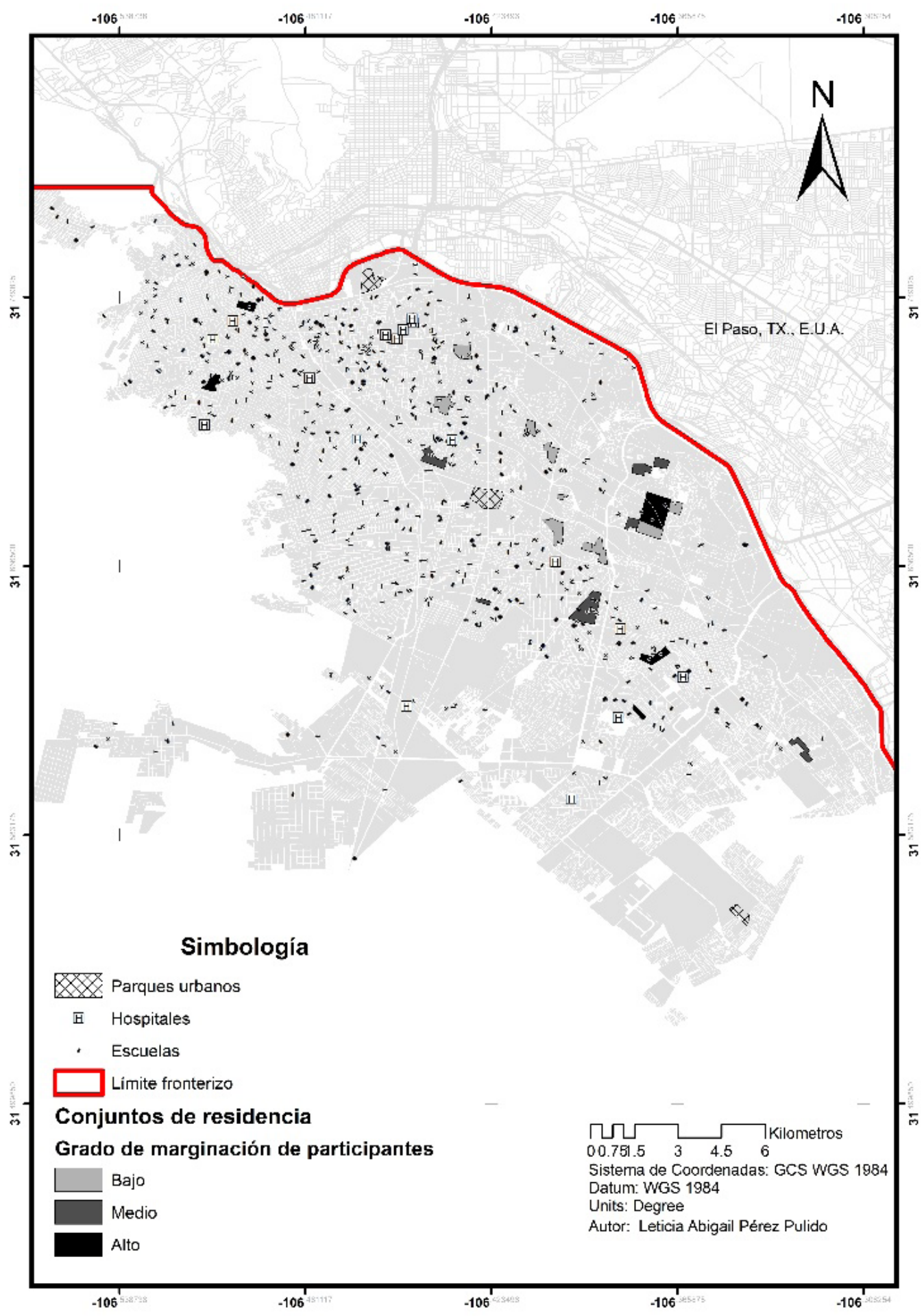

Fuente: Los autores, con datos de INEGI (2010)

Cabe destacar que el grado de marginación de los participantes no refleja el grado de marginación del conjunto habitacional en su totalidad; se reconoce la existencia de 
una multiplicidad al interior de los mismos. Sin embargo, sí muestra la importancia del acceso al equipamiento en personas con un alto grado de vulnerabilidad. En la Tabla 1 se muestra el nivel de percepción de los participantes respecto a su satisfacción por el conjunto habitacional donde habitan, las áreas verdes existentes en su entorno y el nivel de criminalidad, relacionado con los tipos de expresiones de violencias vividas o conocidas. En ella se observa la percepción del entorno expresada por la población en una escala de 1 a 10, siendo 1 el nivel más bajo y 10 el nivel más alto de aprobación del contexto donde habita, así como los porcentajes asignados a la calidad de áreas verdes y la percepción de la criminalidad.

Tabla 1: Percepción de entorno

\begin{tabular}{l|l|l|l}
\hline Vecindario & Satisfacción por vecindario & Satisfacción por áreas verdes (\%) & Seguridad en el entorno (\%) \\
\hline Abierto & 7.0 & 16.66 & 50.00 \\
\hline Cerrado & 8.5 & 81.81 & 45.45 \\
\hline Promedio & 7.6 & 47.82 & 47.72 \\
\hline
\end{tabular}

Como se puede observar, la percepción de las áreas verdes y su calidad difiere mucho entre un vecindario cerrado y uno abierto. La razón principal es el mantenimiento provisto por parte de los comités vecinales, lo que además disminuye ampliamente los factores señalados como contaminantes por parte de los residentes de conjuntos habitacionales abiertos; entre éstos se encuentra la existencia de escombro y basura. Por otro lado, cabe destacar la semejanza en la percepción de seguridad en el entorno, aunque ambos tipos de vecindarios se encuentran en un porcentaje similar, en los vecindarios abiertos acorde a lo expresado por los entrevistados, se puede visualizar una normalización de delitos menores como el vandalismo, robo de automóviles y sus partes e incluso robos a casa habitación. Aún con estas circunstancias, la mayoría de los habitantes de los conjuntos abiertos determinan que los delitos están a la baja, al compararlos con otros de alto impacto que se dieron en años anteriores en su zona, razón por la que ahora se sienten más seguros. Por el contrario, en los fraccionamientos cerrados las personas afirman sentirse seguras porque no han percibido ningún acto delictivo en su entorno inmediato.

Un segundo elemento de análisis fueron las diferencias en relación con la movilidad para llevar a cabo actividades de recreación y esparcimiento, dentro y fuera de sus conjuntos habitacionales (Tabla 2).

Tabla 2: Accesibilidad a las áreas de recreación y de servicios comerciales

\begin{tabular}{l|l|l}
\hline Vecindario & $\begin{array}{l}\text { Disponibilidad de áreas verdes frente a } \\
\text { domicilio (\%) }\end{array}$ & $\begin{array}{l}\text { Dependencia del automóvil para acceder a } \\
\text { zonas comerciales (\%) }\end{array}$ \\
\hline Abierto & 16.66 & 16.66 \\
\hline Cerrado & 63.63 & 81.81 \\
\hline Promedio & 40.14 & 49.23 \\
\hline
\end{tabular}

Al considerar el acceso a las áreas verdes, se establece que la configuración al interior de las zonas habitacionales acrecienta la desigualdad urbana de la población. Esto ocurre sobre todo al considerar la escasez de parques urbanos en la ciudad (Fig. 4). En los conjuntos cerrados, las áreas verdes se encuentran presentes en mayor cantidad y con 
mejor calidad, frente a la dificultad de acceso y los espacios en su mayoría residuales y sin vegetación que se denominan como parques en gran parte de los vecindarios abiertos. En un interesante giro, los habitantes de las zonas habitacionales abiertas identificaron fácilmente el tiempo de desplazamiento a los servicios comerciales de manera peatonal, mientras que el $81.81 \%$ de los habitantes de fraccionamientos cerrados identificaron los lugares con base en el uso del automóvil, generando así una disparidad en el acceso y cambios en los patrones de comportamiento de un vecindario a otro. Así, aunque en los fraccionamientos cerrados existe una mejor disposición de espacios públicos al interior, la falta de satisfactores cercanos limita la posibilidad de llevar a cabo sus actividades diarias sin el uso del automóvil.

Un tercer elemento de análisis fueron las diferencias en la percepción al relacionarlas con la cercanía a instalaciones de salud públicas. Se observó una mayor concentración de centros de salud pública en torno a los conjuntos habitacionales abiertos, lo que hace patente que los habitantes de los conjuntos cerrados pertenecientes a estratos medios y altos, en su entorno inmediato, son clientes cautivos del sector privado para la provisión tanto de servicios de salud como educativos (Tab. 3). En términos de educación, la ciudad presenta un aumento en los tiempos y distancias de traslado según se incrementa el nivel educativo (Pérez y Romo, 2019).

Tabla 3: Accesibilidad a servicios de salud y educativos

\begin{tabular}{l|l|l|l|l}
\hline Vecindario & $\begin{array}{l}\text { Identificación de } \\
\text { instituciones de salud } \\
\text { públicas en el entorno } \mathbf{( \% )}\end{array}$ & $\begin{array}{l}\text { Cercanía a instituciones } \\
\text { públicas de educación } \\
\text { primaria } \mathbf{( \% )}\end{array}$ & $\begin{array}{l}\text { Cercanía a } \\
\text { instituciones públicas } \\
\text { de educación } \\
\text { secundaria } \mathbf{( \% )}\end{array}$ & $\begin{array}{l}\text { Cercanía a } \\
\text { instituciones públicas } \\
\text { de educación media } \\
\text { superior } \mathbf{( \% )}\end{array}$ \\
\hline Abierto & 58.33 & 91.66 & 91.66 & 33.33 \\
\hline Cerrado & 9.09 & 27.27 & 9.09 & 0 \\
\hline Promedio & 31,77 & 59.46 & 50.37 & 16.66 \\
\hline
\end{tabular}

Cabe destacar la concentración de los centros de educación superior y preparación técnica, así como servicios de salud pública en la zona norte de la ciudad, esa concentración de servicios en la zona norte contrasta con su grado de densificación poblacional que es mucho menor que en las zonas nuevas del nororiente y suroriente. Lo anterior ha ocasionado una mayor desigualdad para el acceso a los servicios públicos que han sido ubicados sin considerar la localización de la población, su nivel socioeconómico ni su proceso de envejecimiento.

Esta desigualdad al acceso también se presenta en zonas donde existe una aparente mejora de las condiciones de vida, pues como se observa en la Tabla 3, en las zonas habitacionales cerradas existe una escasa identificación de instituciones públicas ubicadas en los contextos inmediatos, lo cual tras corroborarse geográficamente, muestra que existe un déficit de servicios públicos en torno a los nuevos conjuntos habitacionales, la mayoría de los cuales son de tipo cerrado, hecho que como señalan Pérez y Romo (2019) evidencia la falta de consolidación de nuevos subcentros urbanos a medida que la ciudad se dispersa. 


\section{DISCUSIÓN}

La evidencia expuesta en las secciones anteriores da pauta para discutir la especificidad de la desigualdad urbana que se experimenta entre los habitantes de Ciudad Juárez, Chihuahua, México. La desigualdad urbana en Ciudad Juárez es quizá un caso atípico y ello dificulta equipararla con otros estudios sobre el tema que hayan sido realizados en otras ciudades del contexto mexicano o latinoamericano. En el artículo se ha mencionado que el proceso de urbanización ocurrido en Ciudad Juárez acrecentó la desigualdad, particularmente en términos de la interacción social y urbana entre sus habitantes. Dicho proceso de urbanización tuvo tal impacto porque ocurrió en una localidad aislada del resto de México (donde la ciudad mexicana más cercana se encuentra a $350 \mathrm{Km}$ ), rodeada por abundante suelo desértico de poco valor, bajo la tutela de un grupo pequeño de terratenientes poseedores de la mayor parte de ese suelo, y con interés de generar riqueza a partir de él y de la manipulación en la inversión de la obra pública. Estas condiciones contribuyeron a crear la mayor dispersión urbana del país (Instituto Mexicano para la Competitividad [IMCO], 2014), algo completamente injustificable considerando las condiciones del medio físico y demográfico de la ciudad, generando con ello una gran desigualdad urbana.

En este estudio se evaluó la desigualdad a partir de la percepción sobre condiciones del vecindario, movilidad dentro de la ciudad, cercanía a servicios de salud y educación. Derivado de ello, es posible caracterizar la desigualdad urbana en Ciudad Juárez, entre otras cosas, como aquella en donde las distancias a recorrer y el tiempo de traslado son los dos factores más relevantes para crear distinción entre la población local, en donde la falta de una red de transporte pública eficiente o el no poseer un auto segrega al habitante y le impide realizar actividades de la vida diaria, en donde la población de bajos recursos tiene un acceso limitado al goce de áreas verdes, lo cual representa un enorme factor de exclusión social en una región desértica como es el caso en Ciudad Juárez, y en donde las diferencias en el acceso a los servicios de salud y educación públicos están determinados por la posibilidad de contar con recursos materiales y económicos para movilizarse dentro de la ciudad y no tanto por las posibilidades de tener recursos económicos para pagar por esos servicios. Bajo esas circunstancias, la desigualdad urbana se refleja de manera sui generis en Ciudad Juárez porque un elemento que marca la diferencia entre la población local es el grado de posibilidad que tiene o no para desplazarse en la realización de actividades básicas de la vida diaria.

Al comparar las características de la desigualdad urbana de Ciudad Juárez con otros contextos urbanos del país como, por ejemplo, la Zona Metropolitana del Valle de México (ZMVM) se identifican similitudes en la proporción del gasto y tiempos de traslado entre la población que habita las zonas periféricas de ambas ciudades (Alejo, 2018). Sin embargo, si se considera la dimensión y número de habitantes no es justificable que en Ciudad Juárez le tome a su población alrededor de dos horas recorrer un tramo aproximado de $30 \mathrm{Km}$ para llegar a su destino. Otro caso destacado es el de la ciudad fronteriza de Tijuana, Baja California, cuya expansión física también se originó con el surgimiento de asentamientos irregulares por parte de la población migrante llegada a laborar en la industria maquiladora 
(Ruiz y Aceves, s/f). La diferencia es que, en Tijuana debido a sus límites naturales al norte con los Estados Unidos, al oeste con el Océano Pacifico y sobre todo al sur con el municipio de Ensenada, no se encontraron grupos que concentraran las enormes cantidades de suelo para promover la expansión de la ciudad de manera especulativa como sucede en Juárez. Por tanto, se podría concluir que la dispersión física en la ciudad de Tijuana no es un factor de desigualdad urbana entre la población.

En general, pocos estudios en México discuten en concreto la desigualdad urbana, pues la mayoría se enfoca en analizar solamente los aspectos económicos o si abordan los territoriales se realiza desde con un enfoque macroeconómico al analizar la distribución del ingreso como principal causa de segregación territorial, lo cual no permite comparaciones puntuales de esta investigación con otras ciudades. En esta investigación la desigualdad urbana se evaluó como resultado de la percepción de la población sobre el acceso inequitativo a los recursos públicos urbanos.

\section{CONCLUSIONES Y RECOMENDACIONES}

En este trabajo se ha discutido la generación de desigualdad urbana entre la población de Ciudad Juárez como resultado de la promoción de un modelo de urbanización que ha priorizado la expansión física. Algunos de los factores a los que este estudio ha atribuido la aparición de dicha condición fueron al cambio de visión, desde principios de los noventas, sobre el accionar del Estado que introdujo mecanismos de mercado para la prestación y el otorgamiento de bienes públicos, al impulso de políticas públicas que facilitaron la inserción del sector privado en la construcción y provisión de vivienda, a la reducción del Estado como agente de financiamiento, y en contraparte, la consolidación de las instituciones bancarias como los protagonistas para el otorgamiento de créditos de vivienda, a la especulación con el suelo urbano de la ciudad por parte de grupos económicos locales, y a la manipulación en la provisión de vivienda formal en la periferia para aquella población con bajo poder de crédito, y con ello, la producción de sectores urbanos que presentan condiciones de vida urbana cuestionables.

Como resultado de la reflexión y el análisis de la información desarrollada, es posible dar respuesta a la pregunta origen de esta investigación sobre ¿cómo el modelo de urbanización seguido en Ciudad Juárez, Chihuahua acrecentó la desigualdad urbana entre sus habitantes? Ya se han mencionado algunos de los factores que han contribuido a generar desigualdad urbana, pero hay dos elementos que sobresalen para la respuesta a dicha pregunta: la acción gubernamental y la acción del sector inmobiliario local.

Desde el punto de vista de la acción gubernamental, se puede concluir que los lineamientos de planeación y la política pública que fomentó la expansión física de la ciudad son corresponsables del surgimiento de la desigualdad urbana entre la población local. La acción del sector público ha sido tan determinante que, como se mostró en las Figuras 2 y 3, en los últimos 22 años la mancha urbana de Ciudad Juárez se expandió sin necesidad - casi el doble de su tamaño - ocasionando que aproximadamente la mitad 
de la superficie urbana presente muy baja densidad poblacional con 12 o menos habitantes por hectárea. Ello ha provocado al interior de la ciudad una dualidad de la realidad urbana. Esa dualidad de zonas poco pobladas y zonas medianamente pobladas ha tenido como consecuencia que la carga por habitar, trabajar, convivir y transitar por la ciudad sea más alta para los habitantes que más necesitan interactuar dentro de ella y que habitan en las zonas poco consolidadas. En resumen, la acción pública ha fomentado condiciones de mayor desigualdad para vivir en las zonas donde habitan los grupos con menos poder económico, generalmente ubicadas en la periferia.

Desde el punto de vista de la acción del sector inmobiliario, también se puede concluir que las prácticas de especulación y el manejo que los grupos de poder de la región han hecho sobre los cambios de uso del suelo, también han sido corresponsables para acrecentar las condiciones de desigualdad urbana que se experimentan en Ciudad Juárez. Los grupos inmobiliarios han impulsado, avalados por el sector gubernamental, el desarrollo de vivienda asequible dirigida a la población vulnerable económicamente en las zonas periféricas de la ciudad, porque es allí donde los desarrolladores pueden encontrar mayor recuperación de la inversión. En esas zonas el suelo es barato y la construcción es de baja inversión. El desarrollo de proyectos urbanos, por parte de algunos grupos inmobiliarios, en áreas tan remotas se ha traducido en desigualdad urbana por el hecho de que la población que allí se asienta queda expuesta a escasos servicios públicos urbanos y equipamiento, y a un mínimo interés por parte del sector inmobiliario desarrollador y del sector público local, por apoyar en la generación de nuevos subcentros, creando con ello un patrón de zonas desligadas de la mancha urbana con todas las carencias que ello representa.

La debilidad principal de este trabajo se encuentra en la imposibilidad de poder llegar a conclusiones y resultados más generalizables debido a lo limitado de la muestra consultada. No obstante, lo encontrado en el documento permite identificar lagunas de información y líneas para trabajos futuros de investigación que pueden llegar a enriquecer el tema desde diferentes ópticas y disciplinas. Desde el ámbito de la demografía y de la gerontología urbana, será relevante discutir las diferencias entre la desigualdad urbana experimentada por los adultos mayores y entre diferentes grupos generacionales; desde el punto de vista de la sociología, la relación o no entre el grado de desigualdad urbana y el surgimiento y desarrollo de la criminalidad, entre otras.

En general, como ha sucedido en otros contextos urbanos, los estudios sobre el origen y causas de la desigualdad empiezan a ser más recurrentes dentro de la literatura académica. Por ello, es conveniente visualizar otros enfoques de investigación con diferente temporalidad, tales como las limitantes que las condiciones de desigualdad actual generarán para la movilidad social de las generaciones futuras, o el impacto que generarán las condiciones de vida urbana desiguales en el desarrollo económico local. Estos y otros retos quedan pendientes para los estudiosos de una de las problemáticas más severas que ha originado la globalización, como es la desigualdad urbana y, que viene acompañando en diferentes grados desde hace cuatro décadas la vida del urbanita moderno, y en particular, la del habitante de Ciudad Juárez, Chihuahua, México. 


\section{REFERENCIAS}

Alejo V., D. (2018). Repensar la pobreza y desigualdad en México: el espacio urbano. Nexos economía y sociedad, 15: 459. Recuperado de https:/ / economia.nexos.com.mx/?p=1398

Bullard, R. (1993). Residential apartheid in Urban America. Earth Island Journal, 8(4):35-36.

Consejo Nacional de Población. (n/a). Concepto y dimensiones de la marginación. (Cap. 1). México. Recuperado de http:/ / www.conapo.gob.mx/work/models/CONAPO/Resource/1755/1/images/01Capitulo.pdf

Fuentes, C. (2001). Los cambios en la estructura intraurbana de Ciudad Juárez, Chihuahua, de monocéntrica a multicéntrica. Frontera norte, 13(25):95-118.

George, H. (1963). Progreso y miseria: investigación de la causa de las crisis industriales y del aumento de la miseria al aumentar la riqueza: el remedio. Madrid: Fomento de Cultura.

Harvey, D. (1990). Los límites del capitalismo y la teoría Marxista. México: Fondo de Cultura Económica.

Harvey, D. (2009). The Urban Process Under Capitalism: A Framework for Analysis. International Journal of Urban and Regional Research, 2(1-3):101-131. DOI: 10.1111/j.1468-2427.1978.tb00738.x

Harvey, D. (2012). Rebel cities. From the Right to the City to the Urban Revolution. London: Verso.

Harvey, D. (2014). Seventeen Contradictions and the End of Capitalism. London: Profile Books LTD.

Instituto Mexicano para la Competitividad (2014). Índice de competitividad urbana, ¿Quién manda aquí? La gobernanza de las ciudades y el territorio en México. Ciudad de México. Recuperado de https://imco.org.mx/ indice-de-competitividad-urbana-2014-quien-manda-aqui/

Lefebvre, H. (1972a). Reflexiones sobre la política del espacio. In H. Lefebvre. Espacio y Política. El derecho a la ciudad. (pp. 43-62). Madrid: Ediciones 62.

Lefebvre, H. (1972b). La vida cotidiana en el mundo moderno. Madrid: Alianza.

Lefebvre, H. (1974). La producción del espacio. Madrid: Capitán Swing Libros.

Lefebvre, H. (1983). La revolución urbana. Madrid: Alianza.

Lefebvre, H. (1991). Critique of Everyday Life: Introduction. London: Verso. v.1.

Llera, F. (2015a). Procesos urbanos y política urbana. Ciudad e Interacción urbana. Estados Unidos de América: México Center Rice University / Baker Institute for Public Policy/ Colegio Nacional de Ciencias Políticas y Administración Pública AC/ Alianza para el Desarrollo y la Calidad de Vida S.C.

Llera, F. (2015b). Ciudad, Población y Relaciones Urbanas. Ciudad e Interacción urbana. Estados Unidos de América: México Center Rice University / Baker Institute for Public Policy/ Colegio Nacional de Ciencias Políticas y Administración Pública A.C. / Alianza para el Desarrollo y la Calidad de Vida S.C.

Llera, F.; López-Nórez, A. (2017). Contexto fronterizo y desarrollo desigual. Colaboración transfronteriza en Ciudades Gemelas. Lecciones y Retos Ciudad Juárez / El Paso y Frankfurt (Oder) / Slubice. Estados de Unidos de América: Alianza para el Desarrollo y la Calidad de Vida S.C. / Union for Development and Quality of Life Inc.

López-Nórez, A. (2017). Envejecimiento en el lugar en el contexto urbano mexicano: El Caso de Ciudad Juárez. Envejecimiento y contexto ambiental. Desafíos para el adulto mayor en el contexto de Ciudad Juárez / El Paso. Estados Unidos de América: Colegio Nacional de Ciencias Políticas y Administración Pública, A.C. / Alianza para el Desarrollo y la Calidad de Vida S.C. / Union for Development and Quality of Life Inc.

Maycotte, E. (2010). Espacios abiertos y calidad de vida en conjuntos habitacionales organizados en condominio: el caso de la vivienda tipo económico en Ciudad Juárez, Chihuahua. Toluca: Universidad Autónoma del Estado de México, México.

Nel-Lo, O; Goma, A. (2018). Geographies of discontent: urban segregation, political attitudes and electoral behavior in Catalonia. City, Territory and Architecture, 5 (23):1-12. 
Obeng-Odoom, F. (2015). The Social, Spatial, and Economic Roots of Urban Inequality in Africa: Contextualizing Jane Jacobs and Henry George. American Journal of Economics and Sociology, 74(3):550-586.

Pérez, L. (2018). Propuesta de acción pública para el desarrollo urbano sostenible del suroriente de Ciudad Juárez. (Tesis de maestría). El Colegio de la Frontera Norte, Ciudad Juárez, México. https://www.colef.mx/ posgrado/wp-content/uploads/2018/10/TESIS-P\%C3\%A9rez-Pulido-Leticia-Abigail.pdf

Pérez, L.; Romo, M. (2019). Modelo analítico de justicia socioterritorial: implicaciones de la expansión urbana en el desarrollo social. Economía Sociedad y Territorio, 19(61):479-506. https://doi.org/10.22136/ est20191365

PLAN ESTRATÉGICO DE JUÁREZ. (2019). Foro ciudadano: Economía. Juárez 2030. Plan para una ciudad sostenible. México: Plan Estratégico de Juárez, A.C.

Ruiz, B.; Aceves, P. (s/f). Pobreza y Desigualdad Social en Tijuana. El Bordo Magazine. 2. México: Iberoamericana University Foundation. https:/ / uia-foundation.org/wp-content/el-bordo/02/Tijuana-00. php

Santiago, G. (2013). Políticas federales e intervención empresarial de la configuración urbana en Ciudad Juárez, 1940-1992. México: Universidad Autónoma de Ciudad Juárez - El Colegio de Michoacán, A.C.

Stiglitz, J. (2019a). Introduction. People, power and profits. Progressive capitalism for an age of discontent. Allen Lane: Penguin Random House.

Stiglitz, J. (2019b). Toward a More Dismal Economy. People, power and profits. Progressive capitalism for an age of discontent. Allen Lane: Penguin Random House.

Data de submissão: 19/ jun./2020

Data de aceite: 08/ago./2020 